\title{
3 bài đáng nhớ của ISR năm 2020
}

\author{
Nguyễn Minh Hoàng \\ Hồ Mạnh Toàn \\ Trung tâm ISR
}

Hà Nội, ngày 11 tháng 11 năm 2020

ISR Phenikaa Uni-Khoảng 40 ngày nữa là tới thời kỳ lễ cuối năm. Do đó, thời gian này về cơ bản cũng đủ để xem xét những công việc đã hoàn thành. Đối với các nghiên cứu viên, thời gian vài tháng không nhiều, nhưng cũng cho phép có đánh giá về sản phẩm nào đáng nhớ, để lại dấu ấn của năm.

Trung tâm ISR đóng góp khoảng 30 sản phẩm nghiên cứu đã xuất bản trong 2020. Trong số đó, đáng nhớ nhất là 3 bài đã xuất bản. Những thông số để nói lên tính hợp lý của đánh giá này kèm theo dưới đây.

1. Bài trên Nature: «Reform retractions to make them more transparent » [1]

Nature có tuổi đời 151 năm, là một trong số rất ít ấn phẩm khoa học hàng đầu thế giới, hệ số tác động IF 42.778 (theo JCR 2020).

Do phạm trù đề cập của bài có rất nhiều điểm chạm tới "research integrity", "science publishing", nên đã được dịch, trích lược đăng, giới thiệu lại trên nhiều ấn phẩm khác (ví dụ [24]) bằng nhiều ngôn ngữ quốc tế, trong đó phải kể đến tiếng Đức, Nhật, Trung và Tây Ban Nha.

Đáng nói nhất là bài trích đăng lại trong № 530 của tờ Investigación y Ciencia [2]. Ấn phẩm này là bản tiếng Tây Ban Nha của tạp chí khoa học lâu đời nhất nước Mỹ, năm nay 175 tuổi.

«Investigación y Ciencia es la versión española de la revista norteamericana Scientific American, fundada en 1845 ».

Bài có thể truy cập từ địa chỉ web: https://www.nature.com/articles/d41586-020-01694-x

2. Bài văn hóa, cổ tích và bayesvl: « On how religions could accidentally incite lies and violence: Folktales as a cultural transmitter » [5] 
Nghiên cứu này được coi là một trong những sản phẩm mẫu mực nhất của ISR qua nhiều năm. Trong số khoảng 100 xuất bản phẩm quốc tế của ISR, việc được xem là thuộc số mẫu mực là một đánh giá khắt khe. Nghiên cứu trải qua 3 cái Tết. Trong thời gian làm nghiên cứu đã có lúc phải dừng lại 4 tháng để sản xuất chương trình máy tính bayesvl dùng ngôn ngữ $\mathrm{R}$ [6].

Do sử dụng loại dữ liệu không có tuổi, cho nên các vấn đề rút ra từ kết luận của bài cũng cơ bản là không có hạn chế về thời gian.

Bài có thể truy cập từ địa chỉ web: https://www.nature.com/articles/s41599-020-0442-3

3. Bài COVID-19: «Policy Response, Social Media and Science Journalism for the Sustainability of the Public Health System Amid the COVID-19 Outbreak: The Vietnam Lessons » [7]

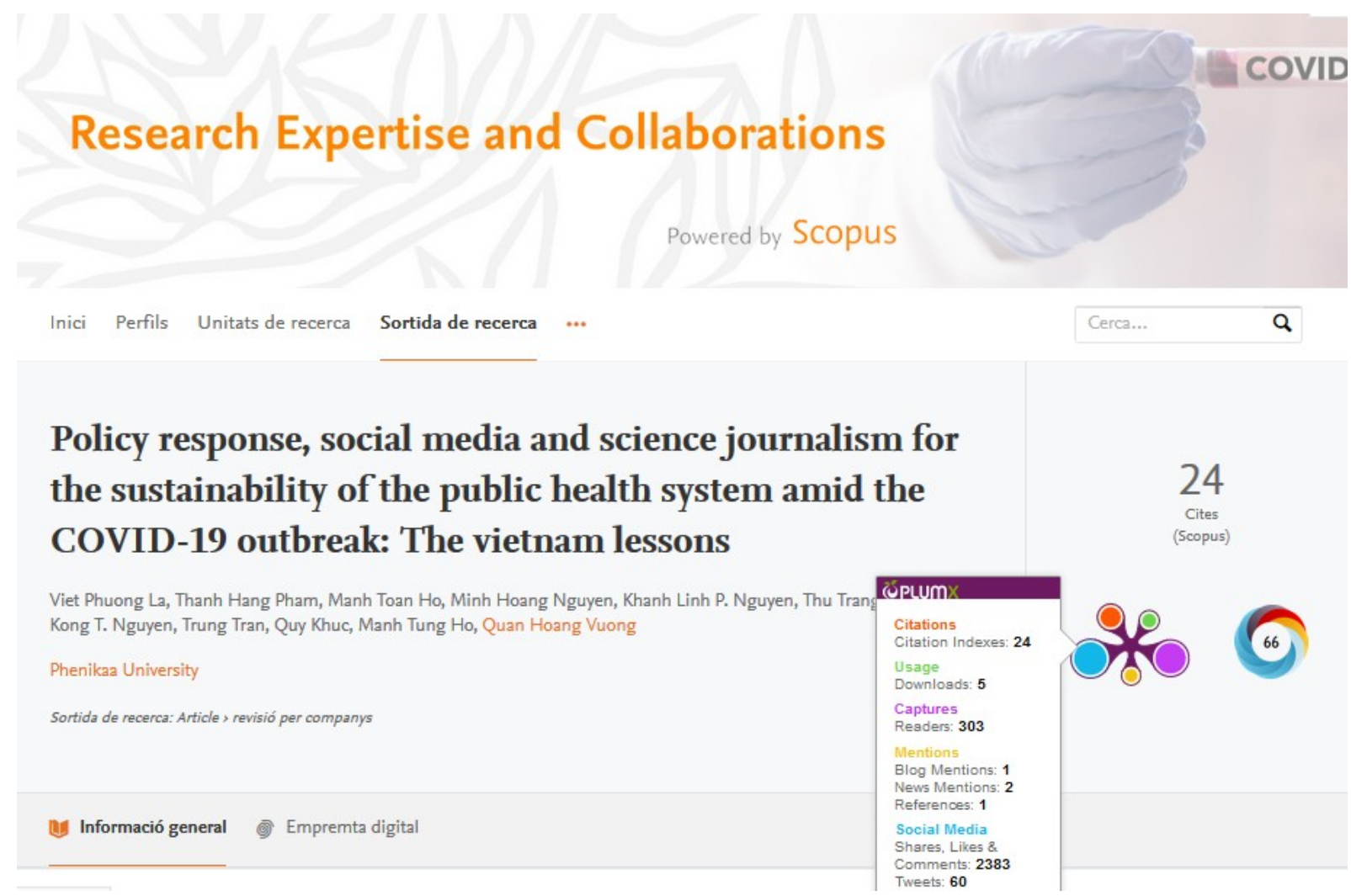

https://covid19.elsevierpure.com/ca/publications/policy-response-social-media-and-sciencejournalism-for-the-susta (accessed: 11-11-2020)

Bài nghiên cứu đã huy động sức mạnh toàn tập thể từ cuối tháng 3-2020, trong lúc áp dụng tình trạng giãn cách xã hội quy mô toàn quốc ở Việt Nam, tình thế bắt buộc trong công cuộc chiến đấu chống dịch COVID-19. 
Đây là một trong những đóng góp đúng lúc, bao quát về phương diện xã hội ngay lúc rất khó khăn. Bài ra đầu tháng 4-2020, và có trích dẫn ISI WOS đầu tiên chỉ 1 tuần sau khi bài xuất bản. Trên trang web của tạp chí, hiện lượng đọc và download bài đã lên xấp xỉ 30.000 lượt chỉ sau 7 tháng. Đây là con số rất lớn. Hàng trăm trang thông tin Internet, bao gồm cả báo chí, các trường đại học, trung tâm nghiên cứu, và nhất là những nguồn thông tin y tế quan trọng nhất trong công cuộc chống COVID-19 đã giới thiệu bài như một nguồn tư liệu.

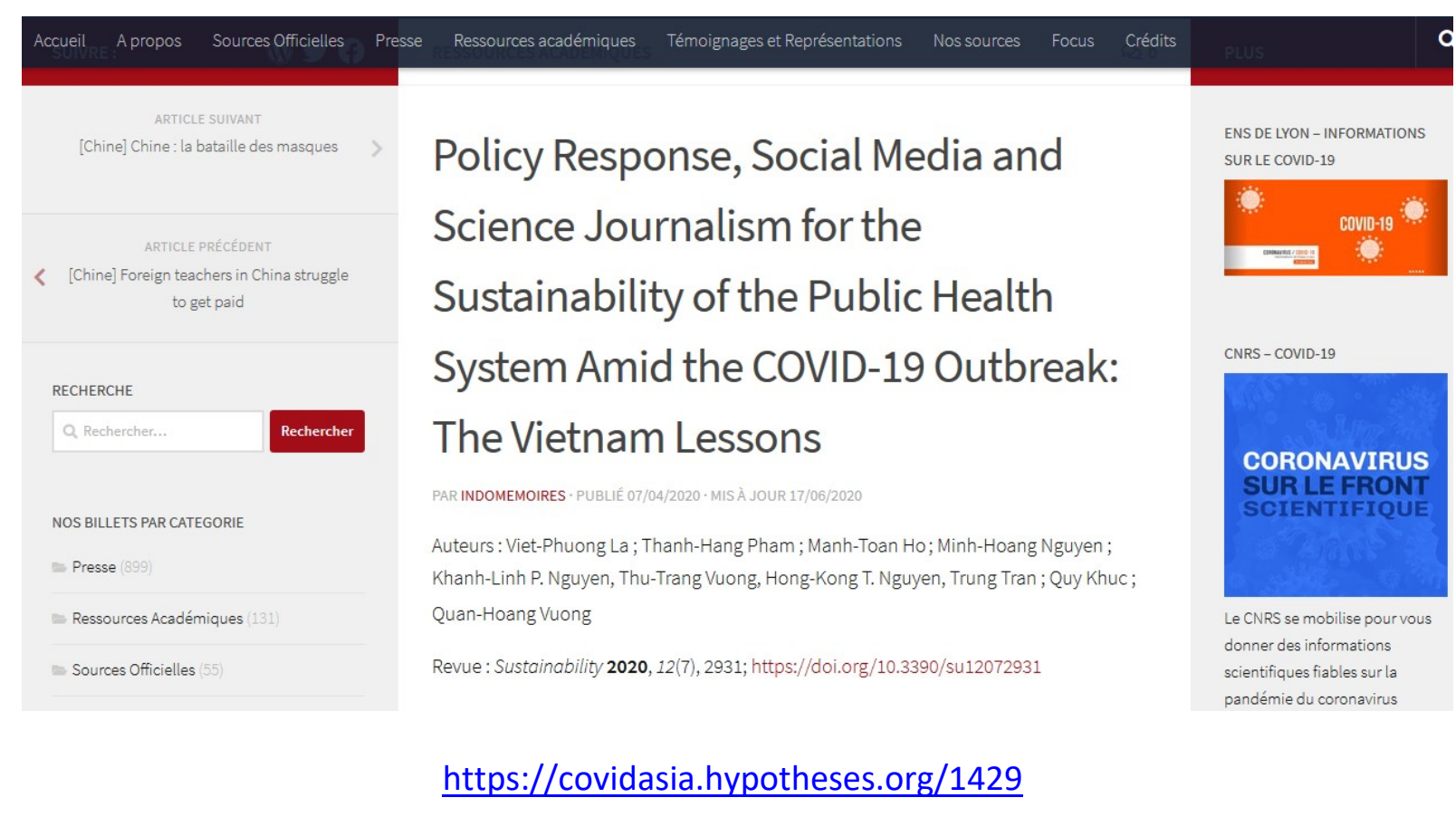

Có thể điểm qua một vài nguồn tiêu biểu trong số này là:

- Exemplars in Global Health (https://www.exemplars.health);

- Elsevier COVID (https://covid19.elsevierpure.com);

- Erasmus University Rotterdam IHS (https://www.ihs.nl/en/journal-articles-covid-19);

- University of Oxford Our World in Data (https://ourworldindata.org/);

- Lawrence Berkeley National Laboratory CovidScholar / US Department of Energy (https://www.covidscholar.org/article/5ec6e6e7d71118fe7dc6b575)

- CNRS Asie Orientale et Coronavirus (https://covidasia.hypotheses.org/1429)

Sau 7 tháng, bài nghiên cứu đã có 80 lượt trích dẫn từ các ấn phẩm nghiên cứu khác, trong đó có 30 từ các ấn phẩm thuộc danh mục Scopus / Web of Science (ISI). Đây là một lượng trích dẫn rất lớn đối với mọi nhà nghiên cứu, đạt được trong khoảng thời gian vài tháng. 


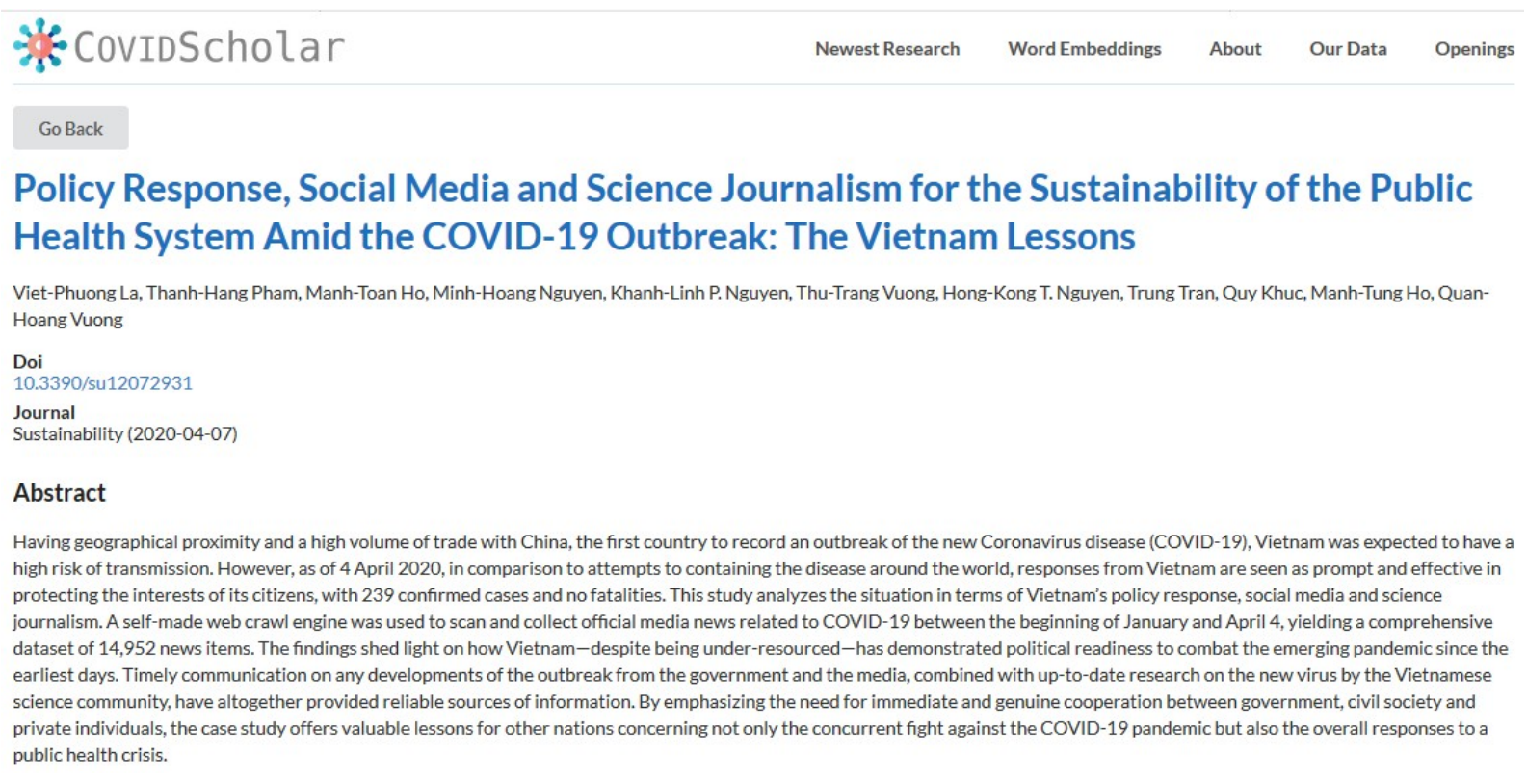

Lawrence Berkeley National Laboratory CovidScholar / US Department of Energy : https://www.covidscholar.org/article/5ec6e6e7d71118fe7dc6b575

Các nguồn media tiêu biểu đã giới thiệu về bài là Project Syndicate, ISEAS-Yusof Ishak Institute, Medium, Asialyst, v.v..

Bài có thể truy cập từ địa chỉ web: https://doi.org/10.3390/su12072931

\section{References}

[1] Vuong, Q. H. (2020). Reform retractions to make them more transparent. Nature, 582(7811), 149.

[2] Vuong, Q. H. (2020). ¿Cómo retractarse en ciencia?. Investigación y Ciencia, № 530 (Noviembre 2020). Retrieved from:

https://www.investigacionyciencia.es/revistas/investigacion-y-ciencia/grandes-hitos-yprximos-retos-de-la-ciencia-811/cmo-retractarse-en-ciencia-19145

[3] Vuong, Q. H. (2020). 論文撤回を改革して透明性を高めょ.Nature ダイジェスト, 17( 8), doi : 10.1038/ndigest.2020.200818. Retrieved from: https://www.natureasia.com/ia$\mathrm{jp} / \mathrm{ndigest} / \mathrm{v} 17 / \mathrm{n} 8 /$ 論文撤回を改革して透明性を高めょ/104106 
[4] Vuong, Q. H. (2020). Retractions: the good, the bad, and the ugly. Nature Index (8 Sept 2020). Retrieved from: https://www.natureindex.com/news-blog/academic-researchpapers-scientists-gain-retractions-the-good-the-bad-the-ugly

[5] Vuong, Q. H., Nguyen, H.-K. T., Ho, M. T., et al. (2020). On how religions could accidentally incite lies and violence: Folktales as a cultural transmitter. Palgrave Communications, $6(1), 82$.

[6] La, V. P., \& Vuong, Q. H. (2019). bayesvl: Visually learning the graphical structure of Bayesian networks and performing MCMC with 'Stan'. The Comprehensive R Archive Network (CRAN). Retrieved from: https://cran.r-project.org/package=bayesvl

[7] La, V. P., Pham, T. H., Ho, M. T., et al. (2020). Policy Response, Social Media and Science Journalism for the Sustainability of the Public Health System Amid the COVID-19 Outbreak: The Vietnam Lessons. Sustainability, 12(7), 2931. 